(NASA-CR-197535) ROSAT DETECTION OF DIFFUSE HOT GAS IN THE EDGE
GALAXY NGC 4631 (COlorado Univ.) $25 \mathrm{p}$
N95-17496

Unclas

G3/89 0034013 
NASA-CR-197535

\title{
ROSAT Detection of Diffuse Hot Gas in the Edge-on Galaxy NGC 4631
}

\author{
Q.Daniel Wang ${ }^{1}$ \\ University of Colorado
}

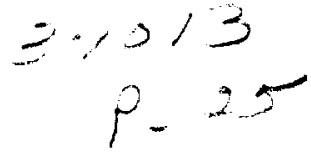

Joint Institute for Laboratory Astrophysics, Center for Astrophysics \& Space Astronomy

Campus Box 440, Boulder, CO 80309

Electronic mail: wqd@rossi.astro.nwu.edu

René A.M. Walterbos ${ }^{2}$ and Michael F. Steakley

New Mexico State University

Astronomy Department, Box 30001/Dept 4500, Las Cruces, NM 88003

Colin A. Norman

Johns Hopkins University \& Space Telescope Science Institute

Department of Physics and Astronomy, 3400 N. Charles Street, Baltimore, MD 21218

and

\section{Robert Braun}

Netherlands Foundation for Research in Astronomy Radiosterrenwacht, P.O. Box 2, 7990 AA Dwingeloo, Netherlands

To appear in the Januray 20 Issue of the Astrophysical Journal

\begin{abstract}
We present our ROSAT observation of the edge-on spiral galaxy NGC 4631, a nearby Sc/SBd galaxy best known for its extended radio halo. Because of the low foreground Galactic X-ray-absorbing gas column density, $N_{H} \approx 1.4 \times 10^{20} \mathrm{~cm}^{-2}$, this observation is sensitive to gas of temperature $Z$ a few times $10^{5} \mathrm{~K}$. We detected soft $(\sim 0.25 \mathrm{keV}) \mathrm{X}$-ray radiation out to more than $8 \mathrm{kpc}$ above the midplane of the galaxy. The strongest $X$-ray emission in the halo is above the central disk, a region of about 3 kpc radius which shows high star formation activity. The X-ray emission in the halo is bordered by two extended filaments of radio continuum emission. We also found diffuse $\mathrm{X}$-ray emission from hot gas in the galaxy's disk. The spectrum of the radiation can be characterized by a thermal plasma with a temperature of $3 \times 10^{6} \mathrm{~K}$ and a radiative
\end{abstract}

\footnotetext{
${ }^{1}$ Post address: Northwestern University, Dept. of Physics \& Astronomy, 2145 Sheridan Road, Evanston, IL 60208-3112.

${ }^{2}$ Visiting Astronomer, Kitt Peak National Observatory, National Optical Astronomy Observatories, which is operated by the Association of Universities for Research in Astronomy (AURA) under cooperative agreement with the National Science Foundation.
} 
cooling rate of $\sim 8 \times 10^{39}$ ergs $\mathrm{s}^{-1}$. This rate is only a few percent of the estimated supernova energy release in the interstellar medium of the galaxy. Analysis of the $\mathrm{X}$-ray spectrum shows evidence for the presence of a cooler (several times $10^{5} \mathrm{~K}$ ) halo gas component that could consume a much larger fraction of the supernova energy. We found strong evidence for disk/halo interaction. Hot gas apparently blows out from supershells in the galaxy's disk at a rate of $\sim 1 \mathrm{M}_{\odot} \mathrm{yr}^{-1}$. This outflow of hot gas drags magnetic field lines up in the halo and forms a magnetized gaseous halo. If the magnetic field lines are still anchored to the disk gas at large disk radii, the outflowing gas may be confined high above the disk by magnetic pressure. We have identified a strong $\mathrm{X}$-ray source which coincides spatially with an $\mathrm{H} I$ supershell. However, the source is likely an extremely luminous X-ray binary with $L_{x}(0.1-2 \mathrm{keV}) \approx 5 \times 10^{39} \mathrm{ergs} \mathrm{s}^{-1}$, which makes it a stellar mass black hole candidate.

Subject headings: galaxies: individual NGC 4631 - galaxies: ISM - galaxies: spiral - X-rays: galaxies

\section{INTRODUCTION}

It has long been theorized that a major, possibly dominant, phase of the interstellar medium (ISM) in the Milky Way is million degree gas created and maintained by supernova explosions (e.g., Cox \& Smith 1974; McKee \& Ostriker 1977). There is, however, no consensus on how much hot gas there might be, and how much of the Galactic disk volume it is expected to occupy (McKee \& Ostriker 1977; Norman \& Ikeuchi 1989; Cox 1990). If sufficiently energetic, the hot gas is expected to break through the cooler gas layers and flow upward, creating a large-scale halo (e.g., Norman \& Ikeuchi 1989) or even leaving the Galaxy as a Galactic wind (e.g., Bregman \& Glassgold 1982; Heckman, Lehnert, \& Armus 1990). However, little observational evidence has been found for either large amounts of hot gas in the Galactic disk, or for the presence of the hot halo. This might be the result of our embedded location in the medium, which hinders derivation of the Galactic-wide properties of the ISM. In particular, the hot diffuse ISM component is predicted to have temperatures up to $\sim 10^{6} \mathrm{~K}$. The resulting soft $\mathrm{X}$-ray emission would be very effectively absorbed by intervening cooler gas. It is also possible that the hot gas may not be as abundant as envisioned in some models. A very hot ISM component with temperatures of $10^{6.3}-10^{7} \mathrm{~K}$ has been discovered in the Magellanic Clouds from analysis of X-ray data from the Einstein and ROSAT X-ray observatories (Wang 1993 and references therein). Furthermore, Bregman \& Pildis (1994) recently presented evidence for several $\times 10^{6} \mathrm{~K}$ gas with a $z$ scale height of $\sim 2.4 \mathrm{kpc}(F W H M)$, similar to that of an extended $\mathrm{H} \alpha$ component, in the edge-on galaxy NGC 891. Here, we report the detection of soft X-ray emission from both the halo and the disk of NGC 4631, another nearby edge-on spiral galaxy of later type. 
NGC 4631 offers many opportunities for us to see what the halo of a spiral galaxy may look like and how it may interact with the disk. The galaxy is located at a distance of only about 7.5 $\mathrm{Mpc}$ (at which $1^{\prime}=2.2 \mathrm{kpc}$ ) and at high Galactic latitude $\left(b=84^{\circ}\right)$ so that absorption of $\mathrm{X}$-rays by Galactic foreground material is small, substantially less than for NGC 891 . This, together with the galaxy's large inclination angle $\left(\sim 85^{\circ}\right.$ with an uncertainty of about $\left.5^{\circ}\right)$, permits direct imaging by the ROSAT of the X-ray emission from million degree halo gas. In addition, NGC 4631, classified as an Sc/SBd galaxy, shows active star formation in its disk, with evidence for disk-halo interactions. In H I, the galaxy is remarkable for its extended tidal tails due to interaction with two neighboring galaries (Weliachew, Sancisi, \& Guélin 1978; Rand \& van der Hulst 1993). This interaction could have triggered the current level of star formation in NGC 4631. Radio observations have revealed a huge radio halo with magnetic field lines radially outward from the galaxy's disk (Hummel et al. 1991). Deep $\mathrm{H} \alpha$ images have uncovered a prominent "double worm" standing above the galaxy's central region, possibly indicating a powerful mass and energy outflow from the disk (Rand, Kulkarni, \& Hester 1992, see also Fig. 1). Our X-ray study reported here provides the direct evidence for this outflow.

\section{OBSERVATIONS AND DATA REDUCTION}

The study was based on a deep (23.5 ks) observation made by the ROSAT Position Sensitive Proportional Counter (PSPC; Trümper 1983). NGC 4631 was covered well within the central 20' radius field of the observation that had a total field of view of $2^{\circ}$ diameter. During part of the observing time, the observation was seriously contaminated by scattered solar X-rays in addition to other noncosmic X-ray events of relatively low intensity levels. To maximize the signal-to-noise ratio for detecting diffuse $\mathrm{X}$-ray-emitting features, we first excluded those time intervals during which the intensity of these contaminating effects was above the cosmic X-ray intensity in the observation. The cosmic X-ray intensity was estimated from the minimum flux in the observation's light curve (e.g., Wang \& McCray 1993).

With the remaining data (totalling $16.8 \mathrm{ks}$ ), we constructed count, background, and exposure images in each of the three PSPC pulse height-invariant (PI) channel intervals: 20-41, 52-90, and 91-201. (The PI binning uses the pulse height energies of individual events recorded by the PSPC and incorporates both the instrument temporal and spatial gain corrections.) The first channel interval is sensitive to photons primarily in the energy range $0.15-0.3 \mathrm{keV}$, here called the soft band. Outside this range the interval's energy response falls below $20 \%$ of its peak value (Snowden et al. 1994). The second and third intervals, with considerable overlapping energy responses, cover the range of $0.5-2.0 \mathrm{keV}$, here called the hard band. The instrument was not sensitive to X-rays in the $0.3-0.5 \mathrm{keV}$ range and above $2.0 \mathrm{keV}$. Counts below PI channel 20 were not used in the image construction because of their large position uncertainties (of a few arcminutes) caused by electronic ghost images (Nousek \& Lesser 1993). We found that residual noncosmic contaminations in the data were uniform across the field. These noncosmic contaminations were included in two 
separate background images, each corresponding to the appropriate band. Exposure corrections (including flat-fielding) were made with exposure images that were constructed in the two energy bands with the aspect information of the observation and the instrument maps made from the ROSAT all-sky survey data (Snowden et al. 1994). An X-ray intensity image was constructed by dividing the count image minus the background image, by the exposure image. The hard band intensity was the sum of the intensities calculated in the two corresponding channel intervals. This minimized the problem that may be caused by possible spectral differences between the NGC 4631 field and the average sky.

The original coordinate information in the data placed the overall morphology of the hard band X-ray radiation above the disk of NGC 4631 . This is partially due to a pointing error of the X-ray observation and partially to the almost edge-on gas disk of the galaxy. To correct for the pointing error of the observation, we cross-correlated our source list and the APM Northern Sky Catalogue (Irwin, Maddox, \& McMahon 1994). Since the typical pointing error of ROSAT PSPC observations is of order $\$ 10^{\prime \prime}$, we obtained APM objects within $15^{\prime \prime}$ radius around each of detected X-ray sources with off-axis angles $<1^{\prime}$. All these objects were fainter than $R=18.9$ or $B=19.5$, and we used the two brightest blue objects $(B=20.33$ and 19.51) to derive the pointing correction. These two objects appear to be the best candidates for two relatively bright X-ray sources and are most likely background AGNs, judging from their X-ray to optical flux ratios and optical colors. This technique for finding optical counterparts was successfully used in the Einstein Extended Medium Sensitivity Survey (Stocke et al. 1991). Detailed discussion of individual $\mathrm{X}$-ray sources in the PSPC observation and their optical identifications will be given in a later paper. Using the APM positions of the two objects $\left[R . A .=12^{h} 41^{m} 26.60 ; \mathrm{Decl} .=32^{\circ} 39^{\prime} 24^{\prime \prime} 1\right.$ and R.A. $=12^{h} 42^{m} 10^{s} 87 ;$ Decl. $=32^{\circ} 22^{\prime} 55^{\prime \prime} .8(\mathrm{~J} 2000.0)$ ], we obtained our best estimate of the $\mathrm{X}$-ray-to-optical position errors: 3.8 to the east, $5^{\prime \prime} 0$ to the north, and 0.26 anticlockwise rotation around the center of the observation. The latter was at $R . A .=12^{h} 42^{m} 7^{3} \cdot 3 ; \mathrm{Decl} .=32^{\circ} 32^{\prime} 30^{\prime \prime} \cdot 1$ (J2000), compared to the galaxy's center at $R . A .=12^{h} 42^{m} 7: 2 ;$ Decl. $=32^{\circ} 32^{\prime} 24^{\prime \prime}$ adopted in this paper. The rotation error is consistent with the systematic value $0.185 \pm 0.099$ in the same direction discovered by the ROSAT team for other PSPC observations (Kuerster 1993). Correcting for these errors also brings the centroid of a PSPC X-ray source, which is $\sim 30^{\prime}$ off the galaxy's center, closer to the position of a previously identified AGN. This particular object had been suggested to be the optical counterpart of the corresponding Einstein source MS 1239.2+3219 (Stocke et al. 1991). After this correction, a residual shift of the X-ray disk relative to the optical and $H I$ disks remains. We attribute this shift to the absorption effects of the disk $(\S 3)$.

In addition to the $\mathrm{X}$-ray data, we have also obtained images in $\mathrm{H} \alpha$ and other emission lines as well as neighboring continuum with the Kitt Peak $0.9 \mathrm{~m}$ telescope. A full discussion of these data will be given elsewhere. In this paper, we use a deep $\mathrm{H} \alpha+[\mathrm{N} \mathrm{II}]$ image corrected for continuum emission for comparison of the distribution of hot and warm ionized gas in NGC 4631. 


\section{SPATIAL DISTRIBUTION}

To study the spatial distribution of hot gas in NGC 4631, we present in Figs. 1-3 overlays of the X-ray intensity images with the $\mathrm{H} \alpha+[\mathrm{N}$ II], radio continuum, and $\mathrm{H}$ I maps of the galaxy. Discrete sources are indicated in Fig. 3. No dominant X-ray source appears in the galaxy's central region. The brightest source is about 2.5 arcmin west of the center, coinciding in position with one of the brightest H II regions (Fig. 1) and one of the only two H I supershells discovered in the galaxy (Rand \& van der Hulst 1993; see also $\S 5$ ). The other supershell, on the other side of the galaxy, also appears to be associated with a discrete X-ray feature ( $(5)$. Apparently, diffuse radiation can be found throughout the galaxy.

Fig. 1 shows an overall spatial correlation between the soft and hard X-ray intensities and the $\mathrm{H} \alpha$ emission of the galaxy. In the hard band (Fig. 1b), the diffuse radiation extends about $24 \mathrm{kpc}$ (full width - see also Fig. 5b) along the major axis of the galaxy, which is comparable to the extent of the galaxy's $\mathrm{H} \alpha$ emission, and $12 \mathrm{kpc}$ (full width - see also Fig. 4b) along the minor axis. In the soft band, the excess radiation above the local sky background extends above the disk as far as $\sim 12 \mathrm{kpc}$, which is about twice more distant than in the hard band (Fig. 4a). The strongest diffuse $\mathrm{X}$-ray feature appears to be connected to $\mathrm{H} \alpha$ filaments in the central region above the galaxy's disk. This is especially apparent near $R A=12^{h} 42^{m 95} ;$ Decl. $=32^{\circ} 33 ! 5$, where double $H \alpha$ filaments several kpc in length stand out above the disk. Immediately adjacent to this region near $R A=12^{h} 42^{m} 5^{*}$ another filament is visible, which possibly forms a giant shell with the rightmost of the double filaments. The data suggest a picture of outflowing hot gas which is initially confined by (partial) shells or chimneys of compressed cooler gas (e.g., Norman \& Ikeuchi 1989). The $\mathrm{H} \alpha$ emission appears less extended than the soft X-ray emission. However, the $\mathrm{H} \alpha$ map covers only a total vertical range of $12 \mathrm{kpc}$, which may be insufficient to define an accurate zero level. A more detailed discussion of the correlation between the X-ray and $\mathrm{H} \alpha$ emission will be given in a later paper.

Comparison of a $0.15-2 \mathrm{keV}$ band X-ray image with the radio continuum map (Fig. 2; Hummel \& Dettmar 1990) shows a similar vertical extent for both. Particularly intriguing is the possible connection between the $\mathrm{X}$-ray emission and two giant radio spurs in the upper half of the halo. These radio spurs are much longer than the $\mathrm{H} \alpha$ filaments and appear not directly associated with them. However, the bright soft $\mathrm{X}$-ray halo emission is largely contained within the boundaries of the spurs, suggesting that the hot gas may be confined by giant magnetic loops (see $\S 5)$ at high distances above the plane.

In Figs. 3a and $3 b$, the X-ray images in the soft and hard bands are superposed with $H I$ contours of NGC 4631 (Rand \& van der Hulst 1993). The lowest gray-scale level in each of the figures is about $1.5 \sigma$ above the $\mathrm{X}$-ray background in the region. The intensity peaks, most of which are above $2.5 \sigma$, show distinctly different distributions in the two images relative to the galaxy's disk which is outlined roughly by the $\mathrm{H} I$ contour of $5 \times 10^{20} \mathrm{~cm}^{-2}$. In the hard band, most intensity peaks in the halo are likely to be background sources (e.g., AGNs and clusters of 
galaxies). Strong, apparently diffuse emission is clearly associated with the galaxy disk itself. A medium-band (PI channels 52-90) X-ray image (Walterbos et al. 1994) shows that most of the extent along the minor axis in the $0.5-2.0 \mathrm{keV}$ image is due to photons with energies between 0.5 and $1.0 \mathrm{keV}$. By contrast, intensity peaks in the soft band tend to avoid regions near the major axis. Most of these peaks have no counterparts in the hard band and appear to emerge from the central portion of the disk. These X-ray morphological differences in the two bands can be attributed to the softness of $\mathrm{X}$-ray emission from hot gas in the galaxy and X-ray absorption by cooler gas in the galaxy's disk. This explanation is further supported by our spectral analysis of the X-ray data ( $\$ 4)$.

There is a distinct asymmetry in the $\mathrm{X}$-ray intensities between the northern and southern halves of NGC 4631 (Figs. 2-4). This asymmetry is stronger than that of the $1.49 \mathrm{GHz}$ radio continuum emission of the galaxy (Fig. 2; Hummel \& Dettmar 1990). NGC 4631 is a not completely edge-on system, and $\mathrm{X}$-ray absorption may be able to account for the asymmetry. The intensities in both the soft and hard bands drop sharply just below the galaxy's major axis (adopted to be $83^{\circ}$ north to east through the galaxy's center in Figs. 1-3; angular distance = 0 in Fig. 4). Such a drop in intensity is not seen in the intensity distribution along the major axis (Fig. 5). This intensity deficit extends about 2.5 below the axis, compared to the intensity distribution above the axis (Fig. 4). The $\mathrm{HI}$ column density in this area is $\gtrsim 0.5 \times 10^{20} \mathrm{~cm}^{-2}$ (Fig. 3). If this column of $\mathrm{H} I$ gas lies in front of the $\mathrm{X}$-ray-emitting region (i.e., the $\mathrm{H} I$ disk tilted with its near side below the axis), the corresponding absorbing optical depth $\gtrsim 0.4(\epsilon / 0.2 \mathrm{keV})^{-2.6}$ can, at least partially, explain the deficit. The $H$ I disk is exceptionally thick with a scale height of $0.5-1 \mathrm{kpc}$ in the outer galaxy and is significantly distorted due to the tidal interaction with the companion galaxies (Rand 1994; Fig. 3). Therefore, the unabsorbed average distribution of the $\mathrm{X}$-ray emission could be much smoother than the observed distribution in the region below the major axis.

Excluding the data points in the area as marked with squares in Fig. 4, we fitted the observed intensity distributions in the two bands with a uniform background plus a Gaussian function that is centered on the major axis. We use this function to provide a convenient description of the intensity distributions. The fits are satisfactory with $\chi^{2} / \nu=16.3 / 21$ and $28.4 / 45$ in the two bands. The background intensity, the height and standard deviation of the Gaussian function from the fits are $5.3_{-0.4}^{+0.4} \times 10^{-4} \mathrm{cts}^{-1} \mathrm{~s}^{-1} \operatorname{arcmin}^{-2}, 3.5_{-1.5}^{+2.1} \times 10^{-4} \mathrm{cts}^{-1} \mathrm{~s}^{-1} \operatorname{arcmin}^{-2}$ and $2.5_{-1.0}^{+1.5} \operatorname{arcmin}$ in the soft band; the corresponding values in the hard band are $1.8_{-0.2}^{+0.2} \times 10^{-4}, 17_{-2}^{+2} \times 10^{-4}$ and $1.2_{-0.2}^{+0.1}$ (all at the $90 \%$ confidence level). The intrinsic $z$ deviations of the distributions should be slightly smaller because of the broadening caused by the inclination of the galaxy's disk and the point-spread function of the instrument. Hummel \& Dettmar (1990) have simulated the broadening effect on their radio continuum distribution along the minor axis and found that the broadening is less than $20 \%$. A broadening of similar order is also expected for our X-ray distribution because the radio and $X$-ray emissions from the galaxy show more or less similar distributions, and the two data sets have similar spatial resolutions. The most serious uncertainty 
in the X-ray emission distribution is from the undetermined amount of the absorption close to the major axis. In the high disk and halo regions $(z \gtrsim 1 \mathrm{kpc}$ ), our model fits should be a reasonably good description of the distribution.

\section{SPECTRAL ANALYSIS}

In Fig. 6, we illustrate X-ray spectral properties of the disk and the halo. The disk and halo spectra were collected respectively inside and outside the $\mathrm{H} \mathrm{I}$ contour of $5 \times 10^{20} \mathrm{~cm}^{-2}$ in Fig. 3, but both within a $5^{\prime}$ radius from the galaxy's center. A local sky background was subtracted which was estimated in an annulus with an inner radius of $7^{\prime}$ and an outer radius of $14^{\prime}$. Corrections were also made for the mirror vignetting as a function of photon energy and off-axis angle. Discrete sources, as marked in Fig. 3, were removed by excluding data around each source within 1.5 times the $90 \%$ source radius (defined to contain $90 \%$ of the expected counts for a point-like source).

The spectra are shown in Fig. 6, and the parameters of our model fits are listed in Table 1. The halo spectrum was satisfactorily fitted with an assumed single-temperature thermal plasma of solar abundances (Raymond \& Smith 1977; updated by Raymond 1991). There is a problem with this fit, however. The upper limit to the foreground absorption in the fit is $N_{H}=3.3 \times 10^{19} \mathrm{~cm}^{-2}$ which is inconsistent with the expected line-of-sight $\mathrm{X}$-ray-absorbing gas column. The Bell Lab $21 \mathrm{~cm}$ survey (Stark et al. 1992) gives a neutral hydrogen column density measurement along the line of sight of $1.3 \times 10^{20} \mathrm{~cm}^{-2}$. Galactic warm ionized gas (Reynolds 1989) in this part of sky is expected to contribute an additional column of $2.0-3.3 \times 10^{19} \mathrm{~cm}^{-2}$, depending on the ratio of neutral to singly ionized helium in the gas (Taylor \& Cordes 1993; Jakobsen 1989). Contributions of heavier elements to the absorption cross section are negligible in the soft band (Morrison \& McCammon 1983). In addition, the ISM in NGC 4631 may also contribute to the absorption (Fig. 3a). In any case, including all these contributions, the total effective absorbing column density should exceed $1.6 \times 10^{20} \mathrm{~cm}^{-2}$. With this lower limit for the absorption, the single-temperature model can be rejected at the $90 \%$ confidence level; an excess of the observed spectrum above the model is apparent at the low-energy end of the spectrum in Fig. 6a. This excess, if characterized by a second thermal plasma component, indicates the presence of a much cooler ( $\mathrm{a}$ few times $10^{5} \mathrm{~K}$ ) gas component in the halo (Fig. 6b; Table 1). The total luminosity of the halo $\mathrm{X}$-ray emission is $1.8 \times 10^{39} \mathrm{ergs} \mathrm{s}^{-1}$ for the single-temperature fit, and $1 \times 10^{39} \mathrm{ergs} \mathrm{s}^{-1}$ for the hard component of the two-temperature fit.

While the evidence for cool gas in the halo is a potentially very important result, it depends crucially on the assumed foreground $\mathrm{H}$ I column. We independently verified the $\mathrm{H} I$ column density estimate from the recent Dwingeloo H I survey (Hartmann 1994). Data from this survey were carefully corrected for stray radiation using deconvolution techniques. We obtained total $\mathrm{H}$ I columns in a grid of points spaced by 0.5 around the position of NGC 4631. The average $\mathrm{H}$ I column was $1.4 \times 10^{20} \mathrm{~cm}^{-2}$, and the spread in the values was small (about $10 \%$ ). We also checked IRAS images of the area, which show no evidence for drastic variations in the Galactic 
foreground dust emission. There is hence little doubt that the low foreground column implied by a single-temperature fit to the halo spectrum is incorrect, and that a two-temperature fit is more realistic. One further caveat is relevant, though. A two-temperature fit is the simplest option if a one-temperature fit fails, but in reality there is probably a continuous temperature distribution in the halo gas. Although there is not enough signal in the data to warrant fits of higher complexity, the two-temperature interpretation is an oversimplification which gives an indication of what the data imply, but not a conclusive picture. For example, this is the reason we cannot give a good mass estimate of the cool component.

The observed disk spectrum cannot be satisfactorily described by a single-temperature thermal model, but can be fitted well with a thermal model of two-temperature components (Fig. 6c; Table 1). This second fit was obtained by assuming a foreground absorption of $10^{21} \mathrm{~cm}^{-2}$. This value is estimated from the sum of the Galactic foreground and half of the average neutral hydrogen column density in NGC 4631 over the region from which the spectral data were collected. Whereas the spectral shape is not particularly sensitive to the assumed absorption, the uncertainty in the absorption may significantly affect the estimate of the soft component emission measure. For instance, an increase of the absorption by a factor of 2 would increase the estimate by a factor of 4 . The total luminosities of the hard and soft disk components are $5 \times 10^{39} \mathrm{ergs} \mathrm{s}^{-1}$ and $7 \times 10^{39}$ ergs s$^{-1}$, respectively.

\section{DISCUSSION}

We have both spatially and spectrally resolved the X-ray radiation from the galaxy NGC 4631. Whereas the hard disk component of the observed X-ray radiation can be reasonably accounted for by an integrated contribution from discrete sources (e.g., $\mathrm{X}$-ray binaries and young supernova remnants), the soft disk component, having its characteristic temperature similar to the (hard) halo gas temperature, most likely represents the hot ISM in the disk. Based on the spectral models obtained in the last section, we can approximately convert the observed count surface intensities into physical quantities of the hot gas. The best-fit single-temperature plasma model for the halo gas (Table 1) gives an $E M /$ count intensity conversion of $31 \mathrm{~cm}^{-6} \mathrm{pc} /\left(\mathrm{cts}^{-1} \mathrm{~s}^{-1} \operatorname{arcmin}^{-2}\right.$ ) for the soft band, where $E M \equiv \int n_{e}^{2} d r$ (see also the notes to Table 1) stands for the emission measure of the $\mathrm{X}$-ray-emitting gas. The $E M$ can also be translated into an energy intensity in the $0.1-2.0 \mathrm{keV}$ range using a ratio of $6.3 \times 10^{-13} \mathrm{ergs} \mathrm{s}^{-1} \mathrm{~cm}^{-2} \operatorname{arcmin}^{-2} /\left(\mathrm{cm}^{-6} \mathrm{pc}\right)$. The corresponding conversions for the disk are 21 (hard band) and $5.6 \times 10^{-13}$, where the emission measure accounts for only the soft, low-temperature component. Using these conversions, one can, for example, obtain the hot gas emission measure estimates: $\gtrsim 1.2 \times 10^{-2} \mathrm{~cm}^{-6} \mathrm{pc}$ (soft band) and $z 6.3 \times 10^{-3} \mathrm{~cm}^{-6}$ pc (hard band), for regions enclosed by the lowest $(2 \sigma)$ contours (i.e., $4 \times 10^{-4}$ and $\left.3 \times 10^{-4} \mathrm{cts}^{-1} \mathrm{~s}^{-1} \operatorname{arcmin}^{-2}\right)$ in Figs $1 \mathrm{a}$ and $1 \mathrm{~b}$, respectively. In the rest of this section, we discuss issues relevant to the properties of hot gas in the galaxy. 


\subsection{Origin of the Hot Gas in NGC 4631}

The most natural source of the hot gas appears to be the hot ISM heated by supernovae in the galaxy's disk. We find that the total radiative cooling rate of the hot gas in the disk and

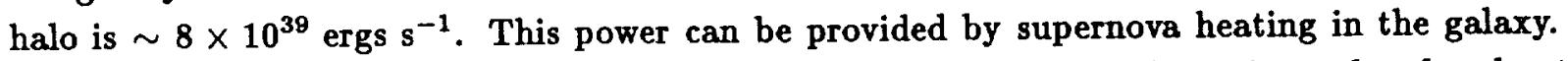
Following Evans et al. (1989), the supernova rate in NGC 4631 can be estimated to be about 0.017 supernovae per year, based on the extinction corrected absolute blue magnitude of the galaxy, taken from Sandage \& Tammann (1981), along with a distance of 7.5 Mpc. This supernova rate estimate represents only a lower limit because the blue magnitude is not a good measure in a galaxy with a dusty central region such as NGC 4631 . The far-infrared luminosity gives a good measure of the massive star formation rate in a vigorously star-forming galaxy such as NGC 4631 and can be used to estimate the rate for Type $\Pi b$ and $I I$ supernovae. Using the far-infrared luminosity from Rice et al. (1988), we derive a Type $\mathrm{Ib}$ and Type II rate of 0.025 to 0.04 supernovae per year, depending on the shape of the initial mass function and the lower mass cutoff. In any case, the supernovae produce ample energy to power the $\mathrm{X}$-ray emission. Adopting a rate of 0.05 supernovae per year, and an energy release of $10^{50}-10^{51} \mathrm{ergs} \mathrm{s}^{-1}$ for each explosion, the total supernova energy input rate is then at least $1.5-15 \times 10^{41} \mathrm{ergs} \mathrm{s}^{-1}$. Therefore, the hot gas cooling rate accounts for about $0.3 \%-3 \%$ of the energy released by supernovae in the galaxy. But, if the cooler halo gas component indeed exists, its radiative cooling could account for a much larger fraction of the supernova energy, $z 1.5 \%-15 \%$ (Table 1 ).

There is evidence for supernova-heated gas flowing out from the galaxy's disk. As mentioned in $\S 3$, two bright X-ray emission peaks $\left(R . A .=12^{h} 42^{m} 23^{s} ; D e c l .=32^{\circ} 32 ! 7\right.$ and $R A=12^{h} 41^{m} 56^{*}$; Decl. $=32^{\circ} 31: 9-\mathrm{J} 2000$ ) in the disk coincide in position with the two supershells (Shell 1 and Shell 2) discovered by Rand \& van der Hulst (1993). These supershells could be the products of numerous supernova explosions in giant $O B$ associations (McCray \& Kafatos 1987). The X-ray peak in the region associated with Shell 1 appears to be extended; the peak is, however, too weak to allow meaningful spectral analysis. Rand \& van der Hulst (1993) pointed out that the shell, which is larger than Shell 2, shows evidence for breakout. So hot gas in Shell 1 may also be blowing out into the halo. Such events are probably more frequently happening in the galaxy's central region where warm gas filaments (or chimneys), at the bottom of the enhanced diffuse soft X-ray feature (Fig. 1; see also Rand \& van der Hulst 1993), are apparently channeling large amounts of hot gas from the disk into the halo. We discuss the nature of the other source in the next section.

We now consider whether the hot gas could be produced by a mechanism other than supernova heating in the galaxy. NGC 4631 is not a typical normal galaxy; the global structure of the galaxy is apparently influenced by the companion galaxy NGC 4656 which has a comparable mass (Rand 1994). Another companion NGC 4627, a dwarf elliptical of low mass, may not have a serious impact on NGC 4631. The tidal interaction between the galaxies is undoubtedly responsible for the large-scale H I morphology in NGC 4631 (Fig. 3). The gas stripped from the galaxy's disk may 
collide with each other or with the disk, and additional velocity disturbances may also be expected in the rotational motion of the disk. Gravitational interactions producing gas collisions or velocity disturbances may lead to shocks. But, the gas heating due to these shocks is unlikely to be able to account for the bulk of the X-ray-emitting gas discussed in this paper. First, the hot halo gas was detected primarily in the galaxy's central region where the tidal interaction should not be important because of the relative deep gravitational potential well of the galaxy itself. Second, the characteristic velocity of the shocks is not expected to exceed $150 \mathrm{~km} \mathrm{~s}^{-1}$, which is the velocity in the flat portion of the galaxy's H I disk rotation curve and also the typical velocity range for the tidal tails (Rand 1994 and references therein). Therefore, the shock could not produce gas of temperature greater than $\sim 3 \times 10^{5} \mathrm{~K}$, too low to account for the hot component of the halo gas. However, it does appear possible that some fraction of the cool halo gas at several hundred thousand degrees may be expected from tidal shocks. However, the sensitivity of the ROSAT data is insufficient to determine if some of the soft X-ray emission above the plane is associated with the $\mathrm{HI}$ tidal tails.

\subsection{Nature of the Brightest X-Ray Source in NGC 4631}

The bright X-ray source coincides spatially with Shell 2. This H I supershell has a diameter of $1.8 \mathrm{kpc}$ and appears to be confined in the disk. The X-ray spectrum of the source can be satisfactorily fitted with either a thermal plasma $\left(\chi^{2} / \nu=23.0 / 25\right)$ or a power law $\left(\chi^{2} / \nu=18.4 / 25\right)$. The constraints on the plasma temperature $>4 \times 10^{7} \mathrm{~K}$ are, however, weak. The foreground absorption is in the range of $0.4-1 \times 10^{21} \mathrm{~cm}^{-2}$ (90\% confidence). If the observed $\mathrm{X}$-ray flux of $1.8 \times 10^{-13} \mathrm{ergs} \mathrm{s}{ }^{-1} \mathrm{~cm}^{-2}$ arises in hot gas enclosed in the supershell, the total thermal energy in the gas would be about a factor of 20 greater than the estimated kinetic energy in the H I shell (6-14 $\times 10^{54} \mathrm{ergs}$; Rand \& van der Hulst 1993). This enormous thermal energy, corresponding to several tens of thousands of supernovae, would have to be supplied by $O B$ associations within the lifetime of the shell $\left(\sim 10^{7} \mathrm{yr}\right)$. This, however, appears to be implausible, and we therefore consider alternative origins for the $\mathrm{X}$-ray source.

The observed intensity of the X-ray radiation from the region coincident with Shell 2 could be dominated by a single super-luminous $X$-ray binary. The power-law fit to the $X$-ray spectrum gives an energy slope of $1.2_{-0.7}^{+1.1}$ and an absorption of $1.3_{-0.6}^{+1.7} \times 10^{21} \mathrm{~cm}^{-2}$. The derived luminosity of the binary, after correction for absorption, is about $5 \times 10^{39} \mathrm{ergs} \mathrm{s}^{-1}$ in the $0.1-2.0 \mathrm{keV}$ band. This luminosity requires a primary mass of $\chi 30 M_{\odot}$, assuming an Eddington limit appropriate for pure electron scattering. Therefore, this $\mathrm{X}$-ray source is likely to be a black hole candidate.

While such X-ray sources are not uncommon in nearby late-type galaxies (e.g., Long \& Van Speybroeck 1983), the source in NGC 4631 is one of the brightest. The observed spectrum might, however, be contaminated significantly by contributions from sources other than the X-ray binary in the region. The complexity of the region is probably similar to that in the 30 Dor area of the Large Magellanic Cloud where X-ray luminous young supernova remnants and superbubbles of hot 
gas as well as a bright massive X-ray binary LMC X-1 are clustered on a scale of $\sim 1 \mathrm{kpc}$ (Wang et al. 1991; Wang \& Helfand 1991). This clustering effect could also make the variability of the binary difficult to detect. The ROSAT data, with their admittedly limited statistics, do not show evidence for X-ray variability, and the PSPC flux is also in reasonable agreement (within a factor of $\sim 30 \%$ ) with Einstein data (Fabbiano \& Trinchieri 1987). An ASCA observation planned by a Japanese team will hopefully provide crucial information on the nature of this intriguing source.

\subsection{Mass Outflow Rate from the Disk}

A crude estimate of the mass outflow rate of hot gas can be derived as follows. The halo $\mathrm{X}$-ray intensity in Fig. $4 \mathrm{a}$ can be converted into a hot gas emission measure $E M$. This EM is effectively an average over a rectangular cut of $14^{\prime}$ length, parallel to the galaxy's major axis. For example, at $z=0$ the average $E M$ implied by the Gaussian fit is $\sim 1.1 \times 10^{-2} \mathrm{~cm}^{-6} \mathrm{pc}$. Hence the $E M$ integrated over the cut is $E M \times 14^{\prime} \times 2200 \mathrm{pc}$ from which we obtain an average hot gas density as $\left(\overline{n_{e}^{2}}\right)^{0.5}=\left(2.8 \times 10^{-3} \mathrm{~cm}^{-3}\right)\left[E M /\left(\delta_{0.1} 1.1 \times 10^{-2} \mathrm{~cm}^{-6} \mathrm{pc}\right)\right]^{0.5}$, where $\delta_{0.1}$ (in units of $10 \%$ ) has been used to characterize the fraction of the disk area that participates in the outflow. Assuming a temperature of $\sim 3 \times 10^{6} \mathrm{~K}$ leads to an estimate of the outflow velocity of the order of the sound speed, $\sim 280 \mathrm{~km} \mathrm{~s}^{-1}$. We can then estimate the mass outflow rate to be about $\sim\left(1.4 \mathrm{M}_{\odot} \mathrm{yr}^{-1}\right)\left(\delta_{0.1} E M / 1.1 \times 10^{-2} \mathrm{~cm}^{-6} \mathrm{pc}\right)^{0.5}$. This estimate probably represents the maximum mass outflow rate in the disk; the average emission measure decreases with increasing angular distance away from the major axis in the $z$-direction (Fig. 4), presumably due to a decreasing area fraction and/or electron density. If the area fraction is approximately constant, the dispersion of $\sim 2.5$ in the intensity distribution fitted to a Gaussian (Fig. $4 ; \S 3$ ) corresponds to a Gaussian dispersion of $\sim 11 \mathrm{kpc}$ in the electron density distribution along the minor axis.

\subsection{Galactic Corona or Galactic Wind?}

The gravity of NGC 4631 alone may not be able to prevent the hot gas from leaving the cold and warm gas disk to form a galactic wind. From the rotation velocity, we estimate that to escape from the gravitational potential of the galaxy, the gas needs to acquire a velocity of about $340 \mathrm{~km} \mathrm{~s}^{-1}$ at a galactocentric radius of several kpc (e.g., Binney \& Tremaine 1987). For an approximately steady flow along streamlines or magnetic flux tubes, we can convert the escape velocity into a corresponding escape temperature $T_{e s}$ using Bernoulli's theorem (e.g., Shu 1992)

$$
-\frac{v_{e s}^{2}}{2}+\int_{0}^{\rho} \frac{d P}{\rho}=0 .
$$

In the adiabatic case $(\gamma=5 / 3)$, this leads to

$$
\left(\frac{T_{e e}}{10^{6} \mathrm{~K}}\right)=\left(\frac{v_{e e}}{267 \mathrm{~km} \mathrm{~s}^{-1}}\right)^{2},
$$


where $n_{H e} / n_{H}=0.1$ is assumed. The derived escape temperature of $1.6 \times 10^{6} \mathrm{~K}$ is about a factor of 2 smaller than the measured characteristic gas temperature in and close to the disk. Therefore, it may just be possible that the gas could escape from the galaxy.

One interesting possibility that we explore here is that the magnetic field in the galaxy may be strong enough to confine the hot gas in a magnetized corona. Near the galactic plane, the mean gas pressure $\sim\left(2.4 \times 10^{-12} \mathrm{dyn} \mathrm{cm}^{-2}\right) \delta_{0.1}^{-0.5}$ seems to be comparable to, or higher than, that of the magnetic pressure $\sim 2 \times 10^{-12} \mathrm{dyn} \mathrm{cm}^{-2}$ which was estimated from a field strength of $\sim 7 \mu \mathrm{G}$ at the plane (Hummel et al. 1991). Therefore, the overpressured outflowing gas can drag magnetic field lines from the disk radially outward into the halo, explaining the outward morphology of the polarized component of the observed radio continuum emission (Hummel et al. 1991). The observed magnetized halo extends to $\sim 13 \mathrm{kpc}$ off the galaxy's major axis, which is comparable to the scale height of the hot gas. The magnetized outflow of hot gas will lose a substantial fraction of its thermal energy and momentum to the magnetic field, whose expansion may be limited if it is anchored to the denser cold and warm ISM in the disk. In addition, the expanding hot gas will also lose energy when it runs into colder gas clouds on its way up. The average field strength in the halo is $\sim 5 \mu \mathrm{G}$ (Hummel et al. 1991), corresponding to an average magnetic pressure of $1 \times 10^{-12} \mathrm{dyn}^{-2}$. This pressure is comparable to, or greater than, the thermal gas pressure in the halo (Fig. 4a); the first contour in Fig. 2a corresponds to a thermal pressure value of $2 \times 10^{-12} \mathrm{dyn}^{-2} l_{\mathrm{kpc}}^{-0.5}$, where $l_{\mathrm{kpc}}$ is the effective path length of the X-ray emitting hot gas along a line of sight (in units of $\mathrm{kpc}$ ). Although some of the regions of hot gas may be able to escape from the galaxy creating a mild galactic wind, most of the outflowing gas appears to be confined ultimately at large galactic radius by the magnetic field together with the galaxy's gravitational potential.

The turnover of the magnetized outflow also explains, at least partially, the large random field component in the halo $(z 80 \%)$, particularly in regions $z 4^{\prime}$ off the major axis where the degree of polarization drops off rapidly (Hummel et al. 1991). Initially, little Faraday depolarization is expected in the halo region. The decrease of the polarization at large $z(\approx 8 \mathrm{kpc})$ probably indicates that the bulk of the outgoing stream of hot gas eventually turns into turbulent motion in the halo. Furthermore, hot gas streams can reach to various different $z$ heights and then turn back to the disk. Therefore, the attached magnetic field lines change their directions at various different positions in halo. The different projections of the lines naturally reduce the degree of the polarization of the radio emission.

The tidal interaction with the companion galaxies should also affect halo gas behavior at large galactic radius, but, as mentioned, it is not likely to play a role in driving the initial outflow. In any case, the outflowing hot gas, accompanied by magnetic fields and cosmic rays, and the relatively low gravitational potential of NGC 4631 seem to offer a natural explanation for both the huge radio halo and the soft $X$-ray-emitting gas in regions far away from the disk of the galaxy. 


\subsection{Comparison with Other Galaxies}

A new result in our ROSAT observation is the evidence for soft $\mathrm{X}$-ray emission in the halo of NGC 4631 with temperatures well below $10^{6} \mathrm{~K}$. Such emission, however, is not at all unexpected. Ultraviolet absorption line studies of the Galactic halo (Savage, Sembach, \& Cardelli 1994 and references therein) clearly indicate the presence of highly ionized species, such as Si IV, C IV N V, and $O$ VI (Davidsen 1993 and Davidsen et al. 1994) which require gas temperatures up to a few times $10^{5} \mathrm{~K}$. With a pressure drop by a factor of $10^{2}$ from the disk to the halo of NGC 4631, adiabatic cooling alone can result in a temperature drop by a factor of 6 , providing a natural explanation for the presence of a few times $10^{5} \mathrm{~K}$ gas in the halo. In addition, gas of temperatures in the correct range can be produced at interfaces of hot gas and cold (or warm) gas clouds through conduction, turbulent mixing (Slavin et al. 1993), or shocks (e.g., Heckman, Lehnert, \& Armus 1990). Our data lack the resolution and sensitivity to determine the exact spatial distribution of the soft and hard components of the hot gas, but it is clear that the radiation is most extended in the soft band.

NGC 891 is the only other galaxy that is somewhat similar to NGC 4631 and has shown strong evidence for the existence of hot gas. Bregman \& Pildis (1994) have recently detected X-ray emission apparently from hot gas in NGC 891. The gas appears to have a temperature around $\sim 10^{6.5} \mathrm{~K}$ and a distribution similar to the warm (Ho-emitting) gas in NGC 891. Unfortunately, because of the large Galactic absorption $\left(\sim 9 \times 10^{20} \mathrm{~cm}^{-2}\right)$ along the line of sight to this galaxy, the predominantly $\lesssim 0.3 \mathrm{keV}$ halo emission beyond the cold and warm gas disk, as we have discovered in NGC 4631, could not be detected in NGC 891 even if it were present.

One somewhat frustrating aspect is that NGC 4631 may not be a typical normal spiral because of its recent interaction with its companions. So it might be somewhat speculative to extrapolate our results to other spirals. Although the galaxy is not in an extreme starburst phase, the hot halo gas certainly seems to arise mainly in the central disk region. Near-infrared imagery would thus be very useful to determine the concentration of star formation in the center. On the other hand, it is becoming clear that many galaxies have had recent encounters, with associated increases in star formation. The situation in NGC 4631 may thus not be atypical at all; we just happen to catch it in a phase through which many galaxies have passed or will pass in the future. The cooling time of the hot halo gas is long enough that the gas might be around for a longer time than the duration of the star burst. But there are few, if any, other likely candidates in addition to NGC 4631, which are located in regions with small Galactic foreground absorptions and are close enough for ROSAT to detect the weak soft $\mathrm{X}$-ray halo emission. Therefore, the spatial and spectral information on the hot gas in NGC 4631 will remain particularly valuable for us to understand the hot gas in the disk and halo of our own Galaxy, and spirals in general. 


\section{Summary}

We have used our deep ROSAT PSPC observation, together with radio continuum (1.49GHz), $\mathrm{H}$ I column density, and $\mathrm{H} \alpha+[\mathrm{N} \mathrm{II}]$ maps, to study the nature of the X-ray radiation from NGC 4631 and the properties of hot gas in both the galaxy's disk and halo. Our main results are as follows:

- The ROSAT data exhibit both spatially and spectrally resolved X-ray radiation from the galaxy NGC 4631. The apparently diffuse radiation extends more than $8 \mathrm{kpc}$ above the galactic plane with a characteristic (Gaussian) dispersion height of $\sim 11 \mathrm{kpc}$ for hot gas density.

- Our detailed spectral analysis shows that X-ray emission from the disk of the galaxy can be attributed to both diffuse hot gas and discrete sources, for example, young SNRs and X-ray binaries.

- The characteristic thermal temperature of hot gas in both disk and halo is $\sim 3 \times 10^{6} \mathrm{~K}$. The total X-ray luminosity of the gas is $\sim 8 \times 10^{39} \mathrm{ergs} \mathrm{s}^{-1}$.

- Supernovae in the galaxy's disk can easily account for the outflow of hot gas from the disk into the halo. The disk-halo mass flow rate is approximately $\sim 1 \mathrm{M}_{\odot} \mathrm{yr}^{-1}$.

- It appears that gravity alone cannot prevent the hot gas from flowing out of the galaxy, but the magnetic field, which is initially driven outward by the gas flow, could confine the bulk of the hot gas at large scale heights.

- We found evidence for a substantially cooler (several times $10^{5} \mathrm{~K}$ ) gas component in the halo. The radiative cooling of this component, though not particularly significant in the 0.15 - $2 \mathrm{keV}$ range, could account for a considerable fraction of the supernova energy input into the ISM.

These findings provide important observational foundations for modeling the mass and energy balance and the disk/halo interaction in spiral galaxies.

We thank R. J. Rand for providing the H I data, R. J. Dettmar for the radio continuum map, M. Dahlem, J. Stocke, and B. Wang for comments. We are also grateful to J. N. Bregman, the referee, for suggestions that led to many improvements in the presentation of the paper. This work was supported by grants from NASA (NAG5-1924) and NSF (AST-9123777) to R. Walterbos, from the Hubble fellowship program and NASA (NAG5-2014) to Q.D. Wang, and from NASA (NAG5-1729) to C. Norman. 


\section{REFERENCE}

Binney, J., \& Tremaine, S. 1987, Galactic Dynamics, Princeton University Press, Princeton, New Jersey

Bregman, J. N., \& Glassgold, A. E. 1982, ApJ, 263, 564

Bregman, J. N., \& Pildis, R. A. 1994, ApJ, 420, 570

Cox, D. P. 1990, in The Interstellar Medium in Galaxies, ed: H. A. Thronson, Jr., \& J. M. Shull, Kluwer Academic Publishers, p181

Cox, D. P., \& Smith, B. W. 1974, ApJL, 189, 105

Davidsen, A.F., 1993, Science, 259, 327

Davidsen, A.F., Bowers, C.W., Kruk, J.W., Ferguson, H.C., Kriss, G.A., Blair, W.P., \& Long, K.S., 1994, submitted to ApJ

Evans, R., van den Bergh, S., \& McClure, R. D. 1989, ApJ, 345, 752

Fabbiano, G., \& Trinchieri, G. 1987, ApJ, 315, 46

Hartmann, D., 1994, Ph.D. thesis, Univ. of Leiden, in preparation.

Heckman, T. M., Lehnert, M. D., \& Armus, L. 1990, ApJS, 74, 833

Hummel, E., Beck, R., \& Dahlem, M. 1991, A\&A, 248, 23

Hummel, E., \& Dettmar, R.-J. 1990, A\&A, 236, 33

Irwin, M., Maddox, S., \& McMahon, R. 1994, Spectrum, 2, 14

Jakobsen, P. 1989, in Extreme Ultraviolet Astronomy, ed: R. F. Malina \& S. Bowyer, Pergamon Press, p281

Kuerster, M. 1993, in ROSAT Status Report, No. 67

Long, K. S., \& Van Speybroeck, L. 1983, in Accretion Driven X-ray Sources. ed. W. Lewin\& E. P. J. van den Heuvel, Cambridge University Press, p41

Norman, C. A., \& Ikeuchi, S. 1989, ApJ, 345, 372

McCray, R., \& Kafatos, M. C. 1987, ApJ, 317, 190

McKee, C. F., \& Ostriker, J. P. 1977, ApJ, 218, 148

Morrison, R., \& McCammon, D. 1983, ApJ, 270, 119

Nousek, J. A., \& Lesser, A. 1993, in U.S. ROSAT Science Data Center Newsletter, No. 8, p13

Rand, R. J. 1994, A\&A, 285, 833 
Rand, R. J., Kulkarni, S. R., \& Hester, J. J. 1992, ApJ, 396, 97

Rand, R. J., \& van der Hulst, J. M. 1993, AJ, 105, 2089

Raymond, J. C, \& Smith, B. W. 1977, ApJS, 35, 419; updated by Raymond, J. C. 1991 (informally distributed and installed in the XSPEC software package)

Reynolds, R. J. 1989, ApJL, 339, 29

Rice, W., et al. 1988, ApJS, 68, 91

Sandage, A., \& Tammann, G. A. 1981, A Revised Shapley-Ames Catalog of Bright Galaxies, Carnegie Institution, Washington

Savage, B. D., Sembach, K. R., \& Cardell, J. A. 1994, ApJ, 420, 183

Shu, F. 1992, in Physics and Astrophysics, Vol 2, Gas Dynamics, Mill Valley: University Science Books

Slavin, J. D., Shull, J. M., \& Begelman, M. C. 1993, ApJ, 407, 83

Snowden, S. L., McCammon, D., Burrows, D., \& Mendenhall, J. 1994, ApJ, 424, 714

Stark, A. A., et al. 1992, ApJS, 79, 77

Stocke, J. T., et al. 1991, ApJS, 76, 813

Taylor, J. H., \& Cordes, J. M. 1993, ApJ, 411, 674

Trümper, J. 1983, Adv. Space Res., 2, 241

Walterbos, R. A. M. et al. 1994, in Proc. of First ROSAT Science Symposium, ed: E. Schlegel, $A I P$, in press

Wang, Q. D. 1993, in Star Formation, Galaxies \& the Interstellar Medium, ed: J. Franco et al., Cambridge Univ. Press, p55

Wang, Q. D., Hamilton, T. T., Helfand, D. J., \& Wu, X. 1991, ApJ, 374, 475

Wang, Q. D., \& Helfand, D. J. 1991, ApJ, 379, 327

Wang, Q. D., \& McCray, R. 1993, ApJL, 409, 37

Weliachew, L., Sancisi, R., \& Guélin, M. 1978, A\&A, 65, 37 


\section{NOTES TO TABLE 1}

$I_{a}$ and $I_{u a}$, corresponding energy intensities with and without absorption, were calculated in the energy range $0.1-2.0 \mathrm{keV}$. A distance of 7.5 Mpc to NGC 4631 was assumed in the calculation of the luminosities. Best-fit parameters were used in calculating the intensities and luminosities except for the hard disk component and the soft halo component, where lower and upper limits of the corresponding temperatures were used, respectively. The integrated emission measure was defined as $\frac{1}{4 \pi} \int_{\Omega} E M \mathrm{~d} \Omega$, where the integration is over the solid angle over which the spectral data were collected (see text) and $E M=\int n_{e}^{2} d r$ with $n_{e}$ being the electron density (all in cgs units).

\section{FIGURE CAPTIONS}

Fig. 1: ROSAT PSPC X-ray intensity contour maps of NGC 4631 in the $0.15-0.3 \mathrm{keV}$ band (a) and in the $0.5-2.0 \mathrm{keV}$ band (b) overlaid on the gray-scale image representing the distribution of $\mathrm{H} \alpha+[\mathrm{N}$ II $]$ emission of the galaxy. These two X-ray maps were constructed with a pixel size of $5^{\prime \prime}$ and smoothed with a Gaussian of $F W H M=40^{\prime \prime}$. For the $0.15-0.3 \mathrm{keV}$ band, each contour interval equals $1 \sigma$ with the lowest contour at $2 \sigma$ above the local background $\left(=5.4 \times 10^{-4}\right.$ counts $\mathrm{s}^{-1} \operatorname{arcmin}^{-2} ; 1 \sigma=2 \times 10^{-4}$ counts s$\left.^{-1} \operatorname{arcmin}^{-2}\right)$. For the $0.5-2 \mathrm{keV}$ band, the contour levels correspond to $2,6,10,14,18,22,26,30,38$, and $54 \sigma$ above the local background ( $=1.8 \times 10^{-4}$ counts $\mathrm{s}^{-1} \operatorname{arcmin}^{-2} ; 1 \sigma=1.5 \times 10^{-4}$ counts $\left.^{-1} \operatorname{arcmin}^{-2}\right)$. The $\mathrm{H} \alpha+[\mathrm{N} \mathrm{II}]$ image is a composite of three frames with slightly different noise levels. The white region above and to the right of the middle in the image is due to the elliptical companion NGC 4627, which has a different stellar population than the disk of NGC 4631, so it was oversubtracted in removing the continuum light. One arcminute corresponds to 2.2 kpc.

Fig. 2: Radio continuum contours at $1.49 \mathrm{GHz}$ superimposed on the ROSAT PSPC image constructed in the energy range of $0.15-2 \mathrm{keV}$. The false color intensity is on a logarithmic scale of $7-60 \times 10^{-4}$ counts $\mathrm{s}^{-1} \operatorname{arcmin}^{-2}$. The radio and $\mathrm{X}$-ray maps have a similar spatial resolution of $F W H M=40^{\prime \prime}$. The contour levels are $0.1,0.2,0.4,0.8,1.6,3,5,10,20,40$, and $100 \mathrm{mJy}(\text { beam })^{-1}$.

Fig. 3: ROSAT PSPC X-ray intensity images of NGC 4631 in the $0.15-0.3 \mathrm{keV}$ band (a) and in the $0.5-2.0 \mathrm{keV}$ band (b). The resolution of the X-ray data is the same as in Fig. 1. The gray scales in the two images begin at levels of $8.4 \times 10^{-4}$ counts s$^{-1} \operatorname{arcmin}^{-2}$ (a) and $4.0 \times 10^{-4}$ counts $^{-1}$ arcmin $^{-2}$ (b) which are about $1.5 \sigma$ above the local X-ray background in the two bands. Discrete sources are marked with crosses. The overlaid contours represent the neutral hydrogen column density distribution in the galaxy (Rand \& van der Hulst 1993). Contour levels of the $\mathrm{H}$ I are $0.5,2,5,10,20,40,80 \times 10^{20} \mathrm{~cm}^{-2}$. The spatial resolution ( $\left.F W H M=35^{\prime \prime}\right)$ of the $\mathrm{H}$ I data is comparable to that of the X-ray data. 
Fig. 4: X-ray intensity distributions along the minor axis of the galaxy NGC 4631. The distributions were calculated in the $0.15-0.3 \mathrm{keV}$ band (a) and $0.5-2 \mathrm{keV}$ band (b), in a cut that is perpendicular to the galaxy's major axis and $\pm 7^{\prime}$ wide about the galaxy's minor axis. The contribution from discrete sources as indicated in Fig. 3 was removed. The solid curves represent the best fits to the data with a model consisting of a local background and a Gaussian function centered on the major axis (angular distance $=0$ ) (see text). The data points marked with squares were not included in the fits because of apparent absorption effects of the galaxy's cold gas disk. The Gaussian widths of FWHM $\approx 6 ! 0$ and $2 ! 7$ in the $0.15-0.3 \mathrm{keV}$ and $0.5-2 \mathrm{keV}$ bands are much broader than the size of the instrument point-spread function $(F W H M \approx 0 ! 5)$. The asymmetry between the distribution of $\mathrm{X}$-ray emission on the two sides of the axis, as seen in both bands, might be naturally attributed to the X-ray absorption by cold gas in the galactic disk which is presumably slightly tilted with its near side below the major axis.

Fig. 5: X-ray intensity distributions along the major axis of NGC 4631. The distributions were calculated in a $\pm 5^{\prime}$ wide cut around the major axis. The solid curve represents the best fit to the data in the $0.15-0.3 \mathrm{keV}$ band (a) with a model consisting of a Gaussian function of $F W H M=5 ! 6$ centered at the minor axis (angular distance=0) and a local background (see text). The similar model does not give a satisfactory fit to the data in the $0.5-2 \mathrm{keV}$ band (b). The contribution from discrete sources as marked in Fig. 3 was subtracted. In particular, the dip around $2^{\prime}$ angular distance is caused by the subtraction of the region contaminated by the brightest hard $\mathrm{X}$-ray source in the disk.

Fig. 6: Observed PSPC spectra of the X-ray radiation from the halo and disk of NGC 4631, together with thermal plasma model fits (see also Table 1). Fig. 6a shows two singletemperature fits to the halo spectrum. The dashed and solid histograms represent best-fits of the model with and without the foreground absorption fixed to a value of $1.6 \times 10^{20} \mathrm{~cm}^{-2}$, an amount that is likely present, based on $H I$ and $H$ II information (see text). Fig. 6b shows the two-temperature fit to the halo spectrum, with the same foreground absorption fixed. The two-temperature components are also plotted separately as the dashed (hard) and dash-dot (soft) histograms. Fig. 6c shows the two-temperature fit to the disk spectrum, assuming a foreground column of $10^{21} \mathrm{~cm}^{-2}$. The contribution of the low temperature component is indicated by the dashed histogram. 
Table 1: Results of the Spectral analysis

\begin{tabular}{|c|c|c|}
\hline \multirow[t]{2}{*}{ Parameter $^{\alpha}$} & \multicolumn{2}{|c|}{ Value and $90 \%$ Error } \\
\hline & Disk & Halo \\
\hline \multicolumn{3}{|c|}{ One-Component Model } \\
\hline Best-fit $\chi^{2} /$ d.o.f. & $63.7 / 23$ & $20.7 / 21$ \\
\hline HI Column Density $\left(10^{20} \mathrm{~cm}^{-2}\right)$ & 78 & $0.0^{+0.3}$ \\
\hline Temperature $\left(10^{6} \mathrm{~K}\right)$ & 2.6 & $2.8_{-0.3}^{+0.4}$ \\
\hline Integrated $E M\left(10^{10} \mathrm{~cm}^{-5}\right)$ & 98 & $0.58_{-0.11}^{+0.13}$ \\
\hline$I_{a}\left(10^{-13} \mathrm{erg} \mathrm{s}^{-1} \mathrm{~cm}^{-2}\right)$ & 4.1 & 1.4 \\
\hline$I_{u a}\left(10^{-13} \mathrm{erg} \mathrm{s}^{-1} \mathrm{~cm}^{-2}\right)$ & 238 & 1.4 \\
\hline Total Luminosity $\left(10^{3 \theta} \mathrm{erg} \mathrm{s}^{-1}\right)$ & 330 & 1.8 \\
\hline \multicolumn{3}{|c|}{ Two-Component Model } \\
\hline Best-fit $\chi^{2} /$ d.o.f. & $19.3 / 22$ & $16.4 / 20$ \\
\hline HI Column Density $\left(10^{20} \mathrm{~cm}^{-2}\right)$ & 10 (fixed) & 1.6 (fixed) \\
\hline \multicolumn{3}{|l|}{ Soft Thermal Component: } \\
\hline Temperature $\left(10^{6} \mathrm{~K}\right)$ & $3.5_{-0.8}^{+0.7}$ & $<0.6$ \\
\hline Integrated $E M\left(10^{10} \mathrm{~cm}^{-5}\right)$ & $2.7_{-0.8}^{+0.7}$ & $>2$ \\
\hline$I_{a}\left(10^{-13} \mathrm{erg} \mathrm{s}^{-1} \mathrm{~cm}^{-2}\right)$ & 2.8 & 0.4 \\
\hline$I_{u a}\left(10^{-13} \mathrm{erg} \mathrm{s}^{-1} \mathrm{~cm}^{-2}\right)$ & 6.4 & 5 \\
\hline Total Luminosity $\left(10^{39} \mathrm{erg} \mathrm{s}^{-1}\right)$ & 6.5 & 26 \\
\hline \multicolumn{3}{|l|}{ Hard Thermal Component: } \\
\hline Temperature $\left(10^{\circ} \mathrm{K}\right)$ & $>27$ & $3_{-1}^{+3}$ \\
\hline Integrated $E M\left(10^{10} \mathrm{~cm}^{-5}\right)$ & $>4.8$ & $0.5_{-0.3}^{+0.3}$ \\
\hline$I_{a}\left(10^{-13} \mathrm{erg} \mathrm{s}^{-1} \mathrm{~cm}^{-2}\right)$ & 2.7 & 0.8 \\
\hline$I_{u a}\left(10^{-13} \mathrm{erg} \mathrm{s}^{-1} \mathrm{~cm}^{-2}\right)$ & 4.8 & 1.1 \\
\hline Total Luminosity $\left(10^{39} \mathrm{erg} \mathrm{s}^{-1}\right)$ & 5.4 & 1.1 \\
\hline
\end{tabular}




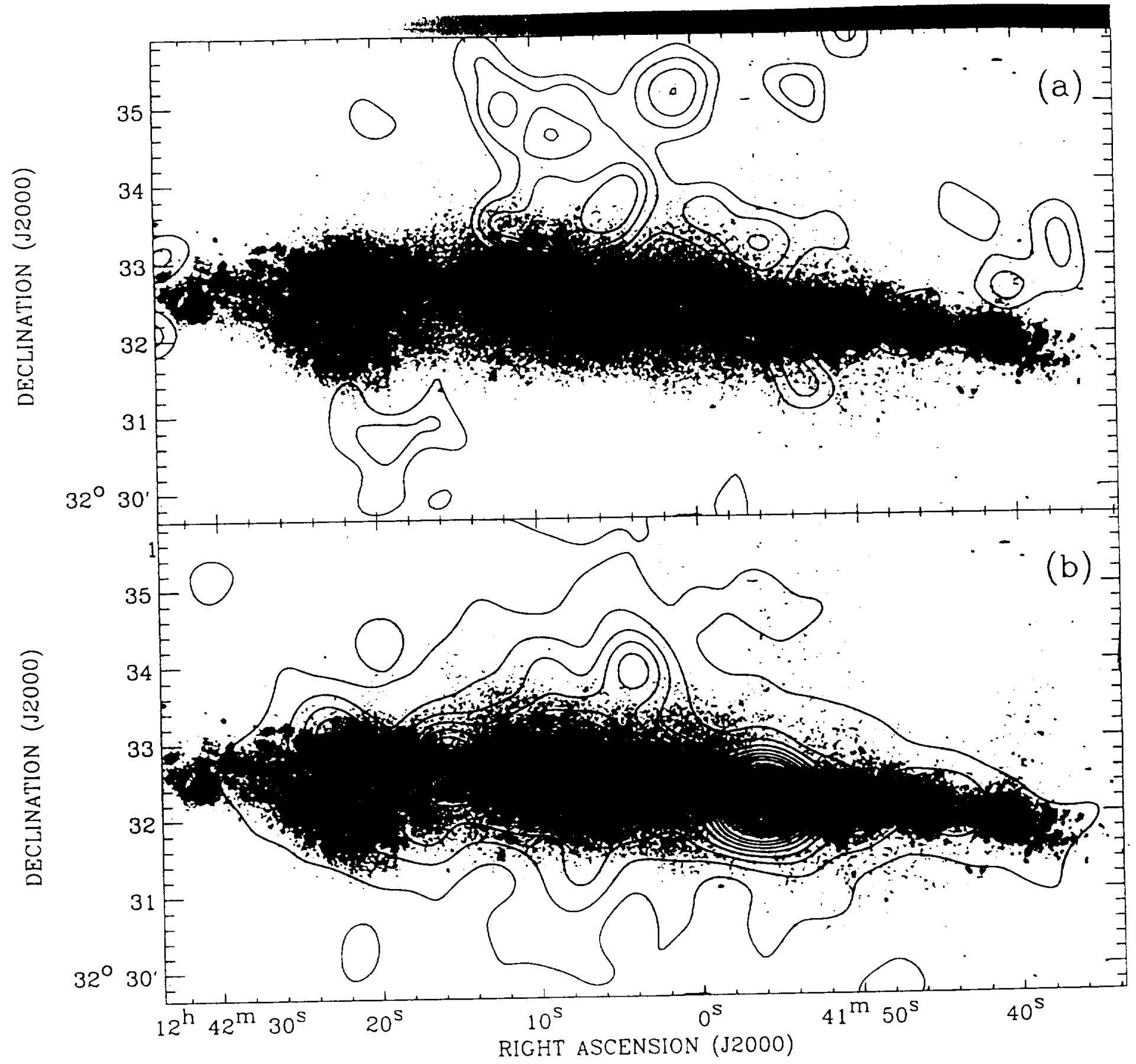

Fig. 1 


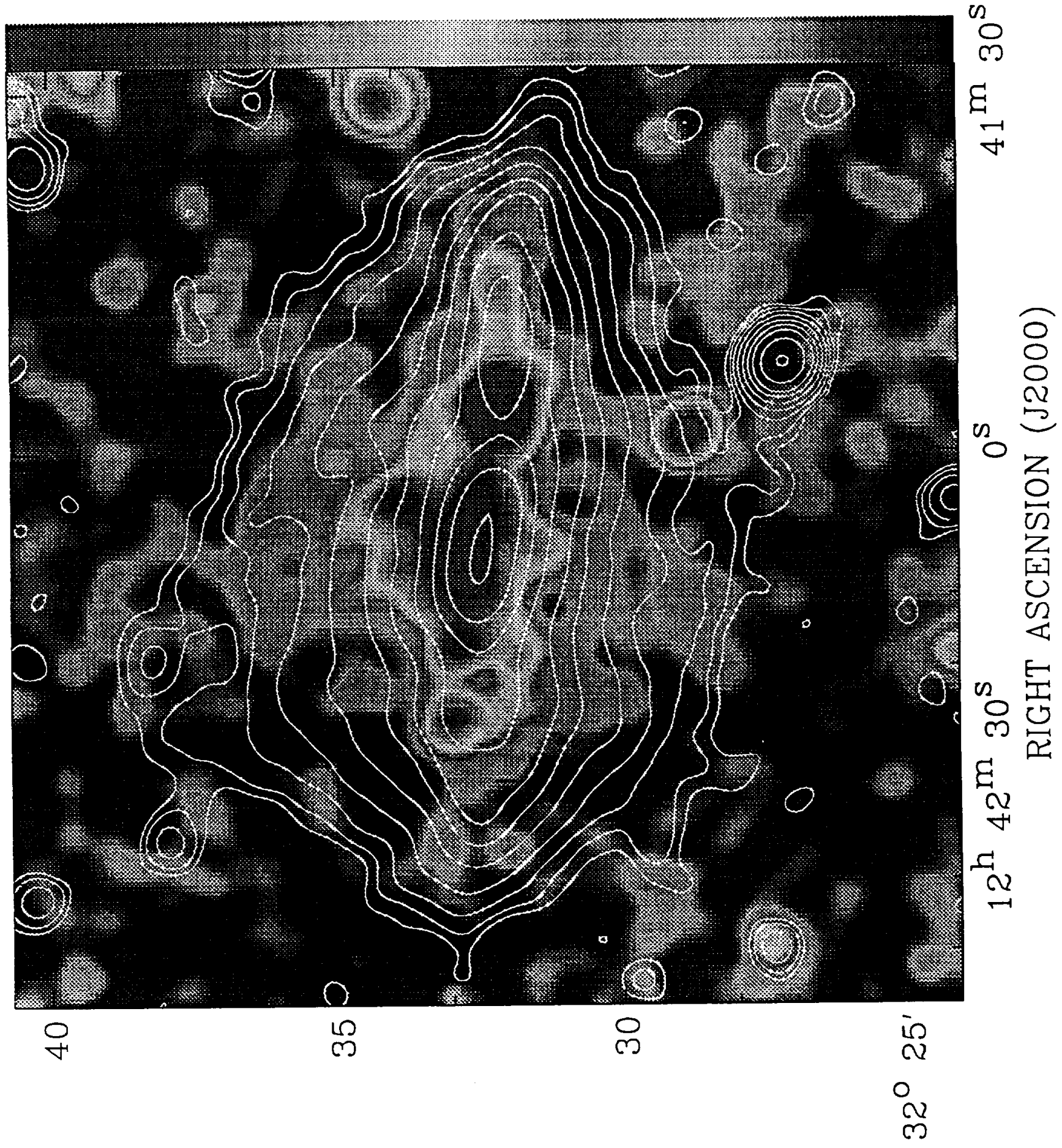

（000Z؟） NOILVNITJHC 


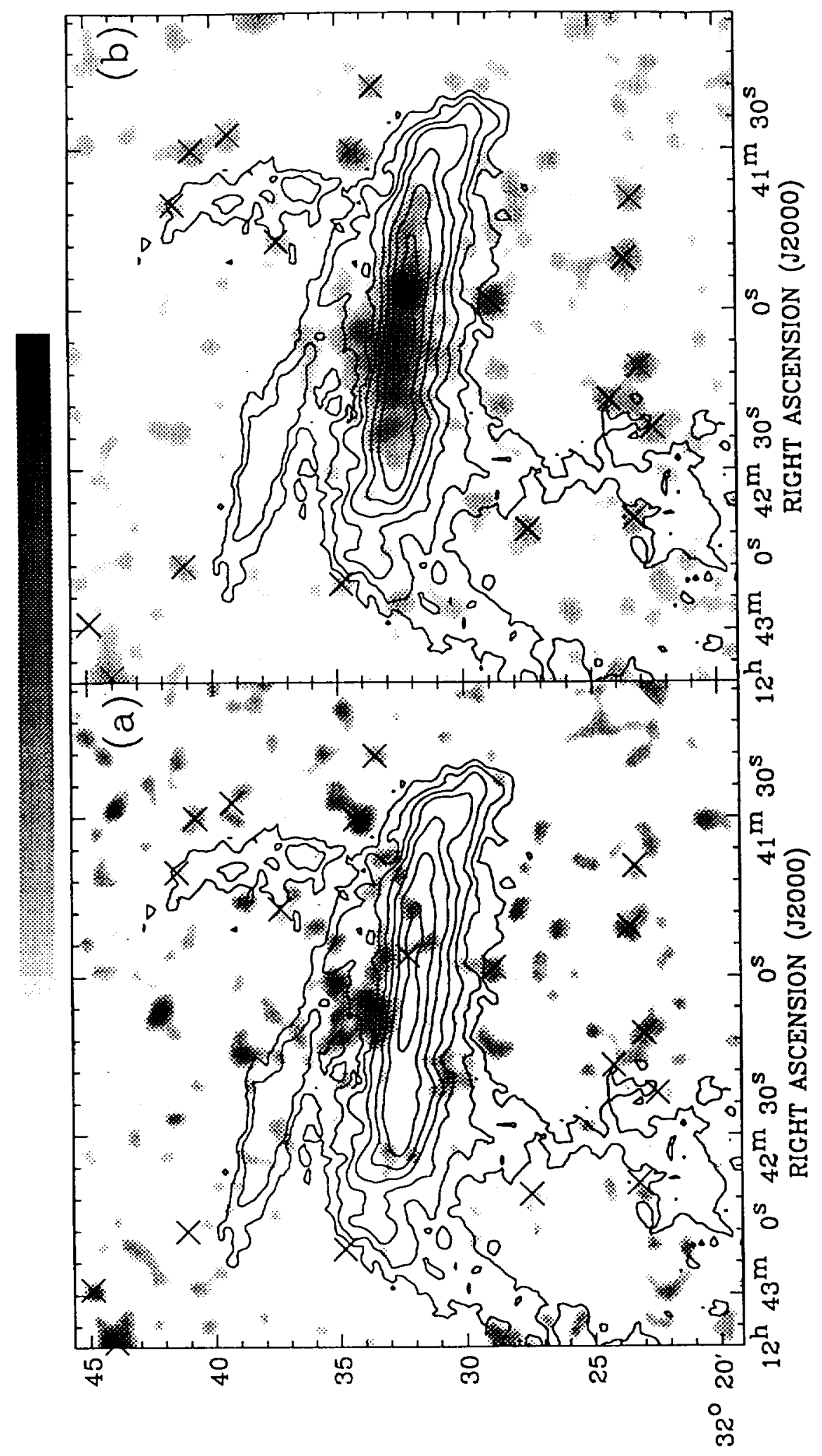

(OOOZ) NOILVNITOGd 


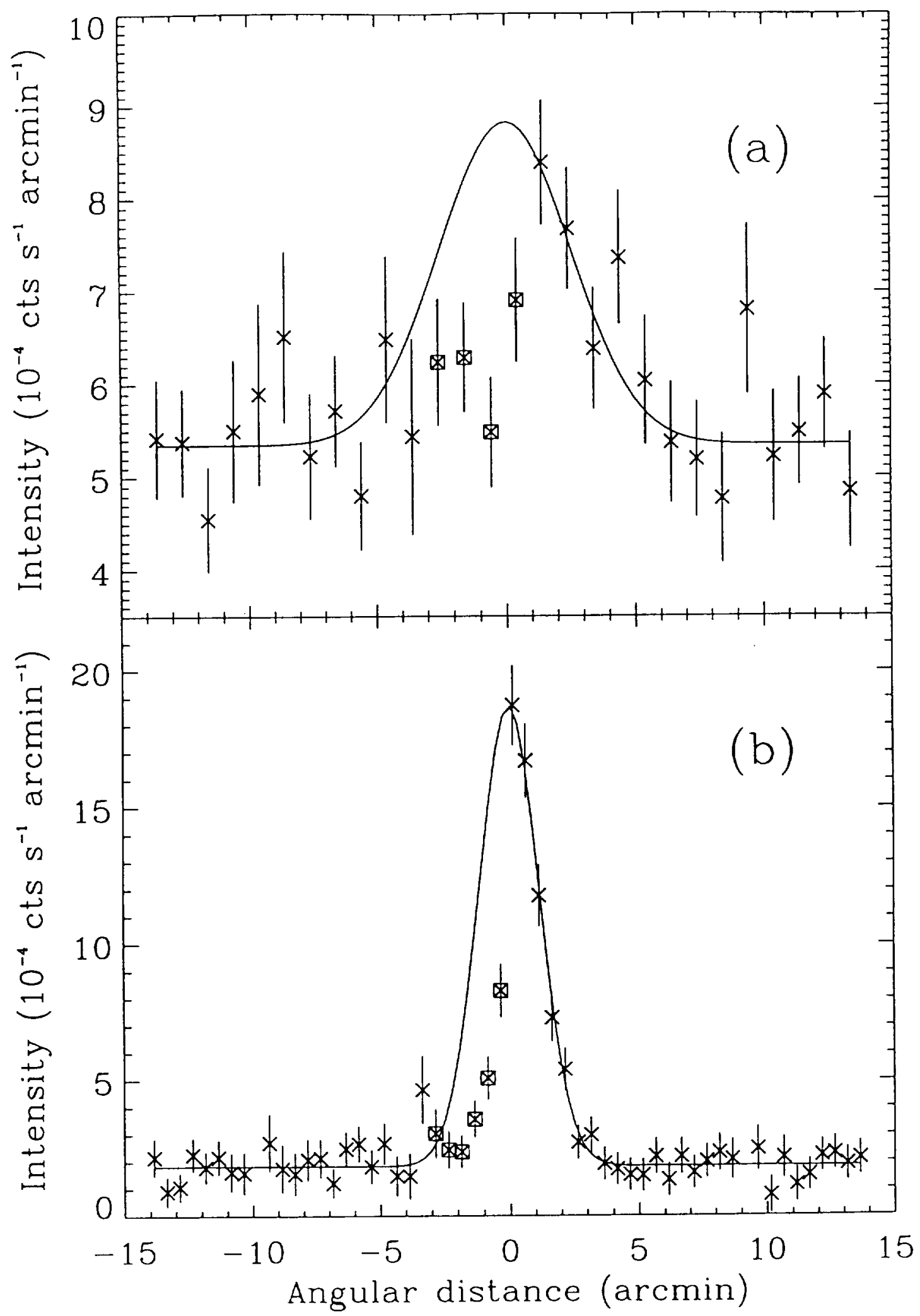

sia 


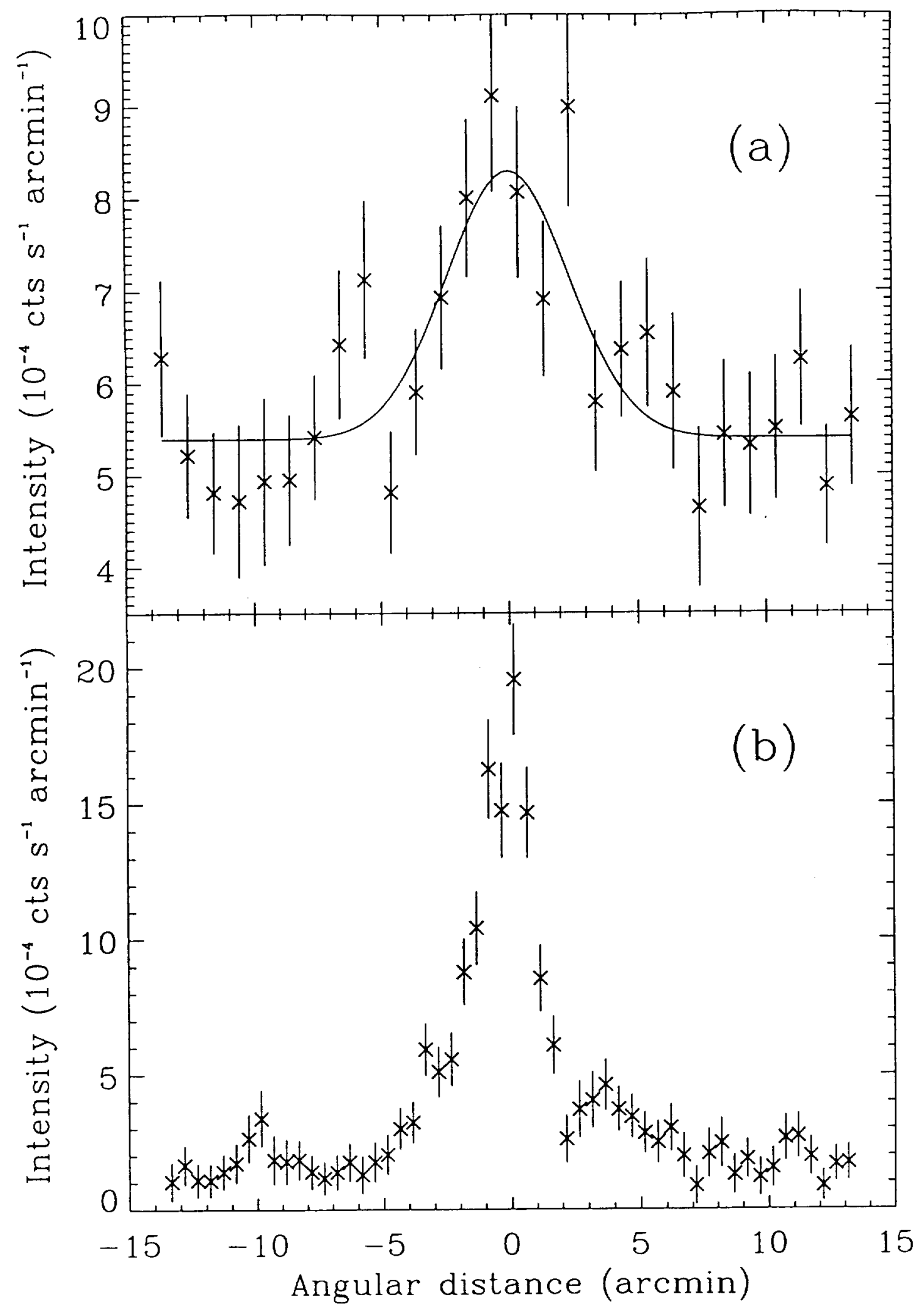

$-5$ 

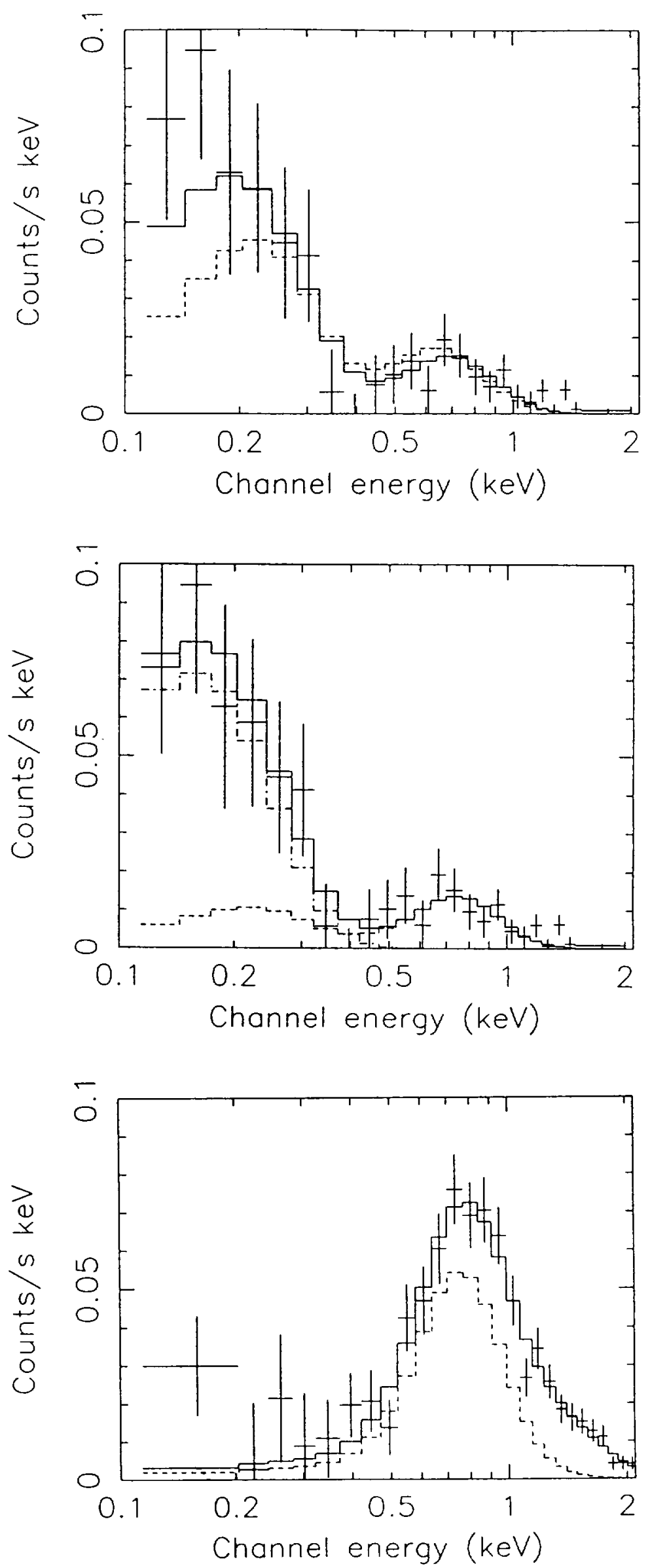

191 